\title{
IgG subclass serum levels in juvenile chronic arthritis违
}

\author{
ALBERTO MARTINI, ${ }^{1}$ ALESSANDRO PLEBANI ${ }^{1}$ ANGELO RAVELLI, $\frac{1}{\mathrm{c}}$ \\ MARIA A AVANZINI, ${ }^{1}$ ROY JEFFERIS ${ }^{3}$ LAURA ZONTA, \\ LUIGI D NOTARANGELO,${ }^{4}$ ALBERTO G UGAZIO,${ }^{4}$ AND \\ ROBERTO BURGIO
}

From the Departments of ${ }^{1}$ Pediatrics and ${ }^{2}$ Genetics and Microbiology, University of Pavia; the ${ }^{3}$ Department. of Immunology, University of Birmingham; and the ${ }^{4}$ Department of Pediatrics, University of Brescia

SUMMARY IgG subclass levels of sera from 26 patients with juvenile chronic arthritis (JCA) were $\stackrel{\circ}{\mathrm{c}}$ determined by means of mouse monoclonal antibodies. Patients were divided into three groups $\mathrm{cr}$ according to clinical activity of the disease: active disease, partial remission, and remission. One $\vec{\circ}$ hundred and sixty four age matched, healthy children served as controls. IgG subclass concentrations were log transformed, and a robust regression method was applied to obtain expected values for the different ages. We found a significant increase of $\operatorname{IgG3}(\mathrm{p}<0 \cdot 0001), \operatorname{IgG} 1 \bar{z}$ $(\mathrm{p}<0.002)$, and IgG2 $(\mathrm{p}<0.035)$ in JCA sera, while IgG4 values did not differ significantly from those of controls. When patients were divided according to clinical activity significant increases of $\vec{\bullet}$ IgG2 and IgG4 were observed in the patients in partial remission. Our data suggest that $\infty_{\sigma}^{\infty}$ differential increase of IgG subclasses during the course of JCA may be of relevance to the pathogenesis of the disease.

Key word: inflammation.

Juvenile chronic arthritis (JCA) is a heterogeneous disease divided into different subtypes on clinical grounds. ${ }^{1}$ Although its causes are unkown, abnormalities in the immune system may be important. ${ }^{2}$ Hyperimmunoglobulinaemia $\mathrm{G}$ is a frequent finding in these patients. ${ }^{3}$ Human IgG consists of four subclasses (IgG1, IgG2, IgG3, IgG4) based on antigenic differences in their heavy polypeptide chains $;{ }^{4}$ each subclass possesses different biological activities, and subclass restriction of the $\mathrm{IgG}$ response to different antigens has been reported. ${ }^{5-7}$ Therefore examination of the patterns of distribution of serum IgG subclass concentrations may provide insight into the immunological process involved in JCA.

In this study we measured the serum levels of $\mathrm{IgG}$ subclasses in patients with JCA by means of mouse monoclonal antibodies specific for each of the four human IgG subclasses. Increase of IgG3 and IgG1 and, to a lesser extent, IgG2 was found; moreover, a relationship was observed between serum levels of some subclasses and clinical activity of the disease.

Accepted for publication 9 October 1985

Correspondence to Professor Alberto Martini, Department of Pediatrics, University of Pavia, Policlinico San Matteo, 27100 Pavia, Italy.

\section{Patients and methods}

\section{PATIENTS}

Twenty six children, 11 boys and 15 girls, ranging in age from 3 to 14 years were studied. All fulfilled the criteria for diagnosis of JCA and were classified according to the type of onset by the criteria of the JRA Criteria Subcommittee of the American $ᄋ$ Rheumatism Association (ARA). ${ }^{8}$ Eight patients presented a systemic form, four a polyarticular응 form, and 14 a pauciarticular form. Patients positive $D$ for HLA-B27 or for rheumatoid factor or with $\operatorname{IgA}$ 을. deficiency were excluded from the study. Disease $\bar{N}$ was defined as active if there was evidence of ${ }^{\circ}$ synovitis on physical examination or if slit lamp $N$ examination showed active iridocyclitis. Remission ${ }_{\mathrm{C}}$ was defined according to the suggested ARAO criteria for rheumatoid arthritis; ${ }^{9}$ those patientso without active joint or eye disease, but not meeting the criteria for remission, were considered to be in $\stackrel{\oplus}{+}$ partial remission. At the time of the study eight 0 patients were receiving no treatment, 10 were taking

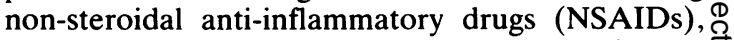
six were receiving oral gold (auranofin) and $\underset{\complement}{\mathbb{Q}}$ NSAIDs, and two were taking prednisone. The $\frac{0}{\sigma}$ results were compared with normal values obtained 
in 164 healthy children ( 89 boys and 75 girls) aged $3-14$, with about 15 in each of these years of age.

\section{QUANTIFICATION OF SERUM}

I M M U NOG LOB U LINS

Serum IgG, IgA, and IgM levels were determined by radial immunodiffusion. ${ }^{10}$

\section{QUANTIFICATION OF SERUM IgG}

$S$ U B C L A S S ES

IgG subclasses were measured by radial immunodiffusion in $1.4 \%$ agarose in $0.1 \mathrm{M}$ barbitone buffer pH 8.6 containing 6\% polyethylene glycol (PEG 3000) (Fluka). Monoclonal antisera to IgG1 (JL 512), IgG2 (GOM 1), IgG3 (ZG4), and IgG4 (RJ4) were diluted in the agarose at concentrations of $0.4 \%$ for IgG1, $0.6 \%$ for IgG2, $0.25 \%$ for IgG3, and $0.8 \%$ for IgG4. ${ }^{11}$ Test sera were diluted $1 / 40$ for IgG1, 1/10 for IgG2, and 1/5 for IgG3 and IgG4 in barbitone buffer. Plates were left at $4^{\circ} \mathrm{C}$ for 48 hours, washed for 24 hours in barbitone buffer containing $1 \%$ glutaraldehyde, and stained with Coomassie brilliant blue. Results were calculated using a serum as standard whose IgG subclass concentrations were calculated from WHO 67/97 serum.

STATISTICAL ANALYSIS

A sample of healthy children aged 2 to 14 ( 89 boys and 75 girls) was used as a reference population. The IgG levels (log transformed) of these children were analysed with a robust regression method to fit separate age dependent curves for each subclass, ${ }^{12}$ and percentiles were calculated based on the deviation of each value with respect to a fitted value specific for age. In all IgG subclasses binomial distribution probabilities were calculated for the group of JCA patients with respect to the percentile values of the reference population. Moreover, in
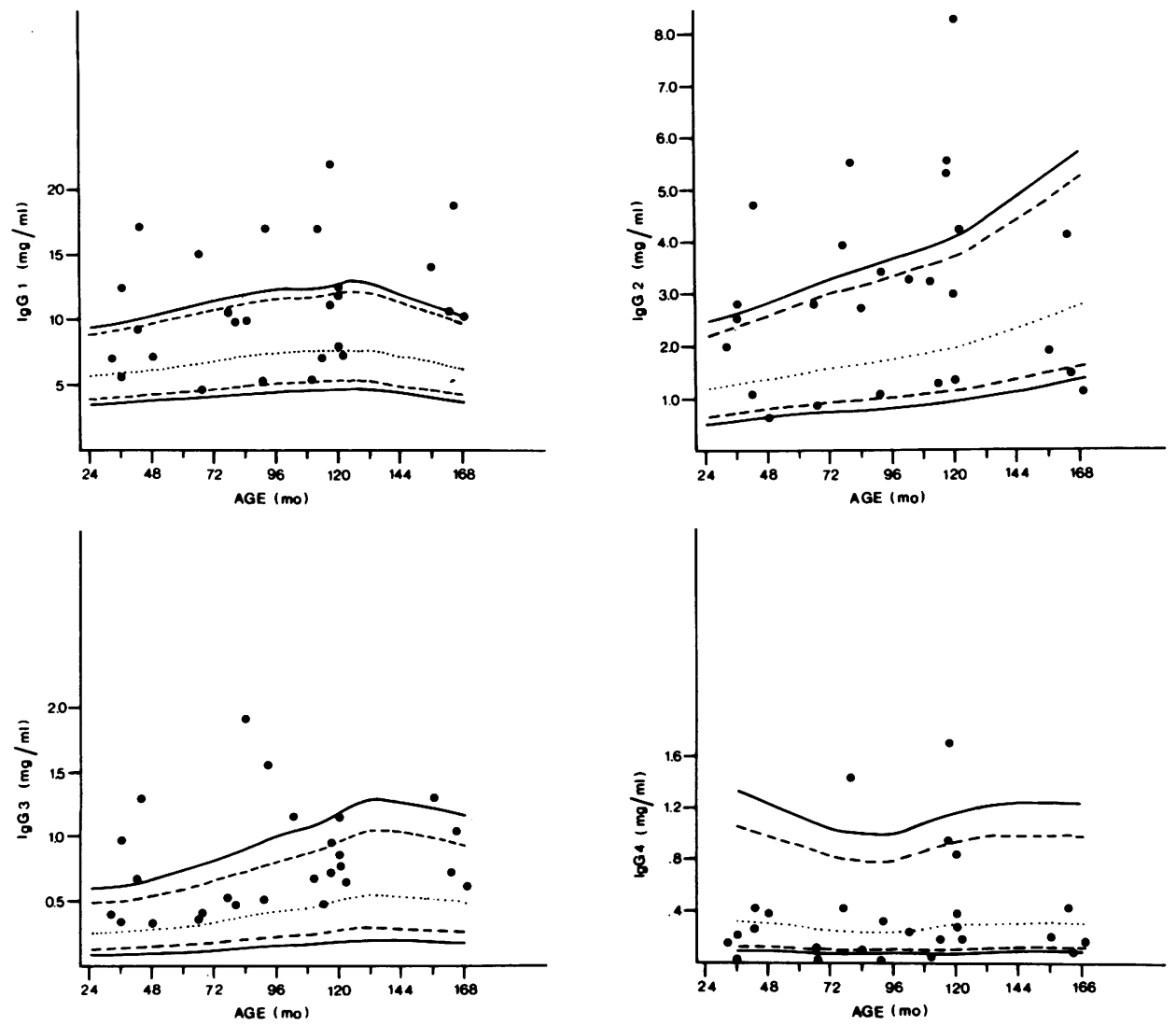

Fig. 1 The four graphs refer to the four IgG subclasses. The age dependent curves are those obtained from a sample of 164 healthy children. The fitted values (.....), the 5th and 95th percentiles (- - ), and the 10th and 90th percentiles (---.-) are shown. The circles represent the concentration levels of JCA individuals. 
order to compare subgroups of JCA individuals taking into account either JCA subtypes, types of treatment, or clinical activity of the disease, for each subject the deviation of the concentration observed from that expected for an individual of the same age was calculated. Distribution of the deviations of the different subgroups was compared statistically by the Mann-Witney non-parametric $U$ test. ${ }^{13}$

\section{Results}

Results of IgG subclass distribution in JCA patients are illustrated graphically in Fig. 1 in comparison

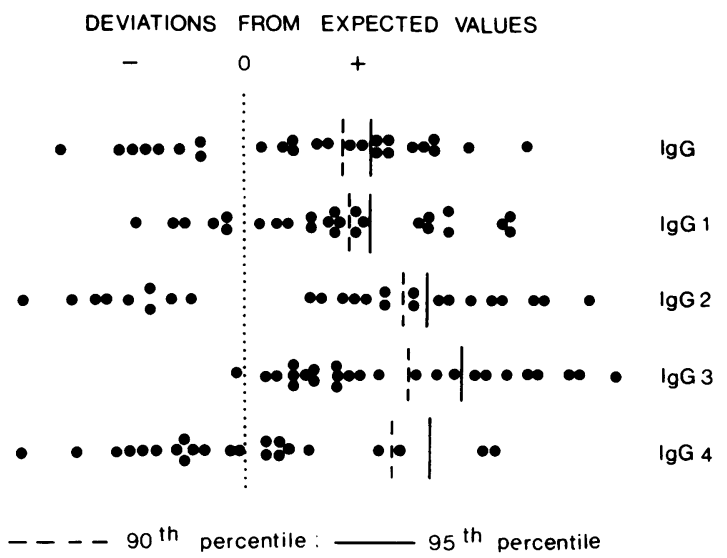

Fig. 2 The values plotted are the (positive or negative) deviation of each JCA patient from the value expected for an individual of the same age. All the values have been log transformed.

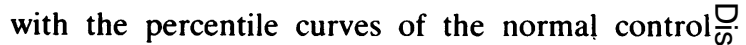
population; in Fig. 2 the data are expressed as deviations from expected values. As expected, the patients presented higher levels of IgG than theo control population $(p<0.017)$; serum levels of IgG흐 were comparable in males and females. Significantly $\frac{\bar{s}}{\vec{b}}$ higher values of IgG3, IgG1, and IgG2 were $\stackrel{\mathbb{\complement}}{\varrho}$ observed, while IgG4 values did not differ signifi-@ cantly from those of controls. Within the $\mathrm{IgG}_{-}$ subclasses IgG3 presented the most marked increase? ( $\mathrm{p}<0.0001)$; all patients but one had values above $\vec{\omega}$ the 50th centile (C). For IgG1 20/26 patients had $\omega$ serum values above the 50th $C(p<0.002)$, while none were below the 10th C. For IgG2 17/26\% patients had values above the 50th $C(p<0.035)$ and one below the 5 th $\mathrm{C}$; this patient had normal levels of IgG4. Serum levels of IgG4 did not differo significantly from those of controls; four patientso presented values below the 5 th $\mathrm{C}$. None of the patients with low IgG2 or IgG4 levels had a personalz or family history of recurrent infections. No correlation was found between serum levels of $\operatorname{IgG} \vec{\bullet}$ subclasses and sex, erythrocyte sedimentation rate, JCA subtype, disease duration, treatment, presence. of antinuclear antibodies, and IgM or IgA serum levels.

When patients were divided into three groups according to clinical activity of the disease (active $\stackrel{2}{\not}$ disease, partial remission, and remission) (Fig. 3) $\stackrel{\mathbb{\Omega}}{\complement}$ the children in partial remission showed significantly $\vec{\overrightarrow{ }}$ higher values of $\mathrm{IgG} 2$ than those with active disease 3 $(p<0.05)$ or than those with remission $(p<0.01)$ and significantly higher values of IgG4 than patients in remission $(p<0 \cdot 025)$.

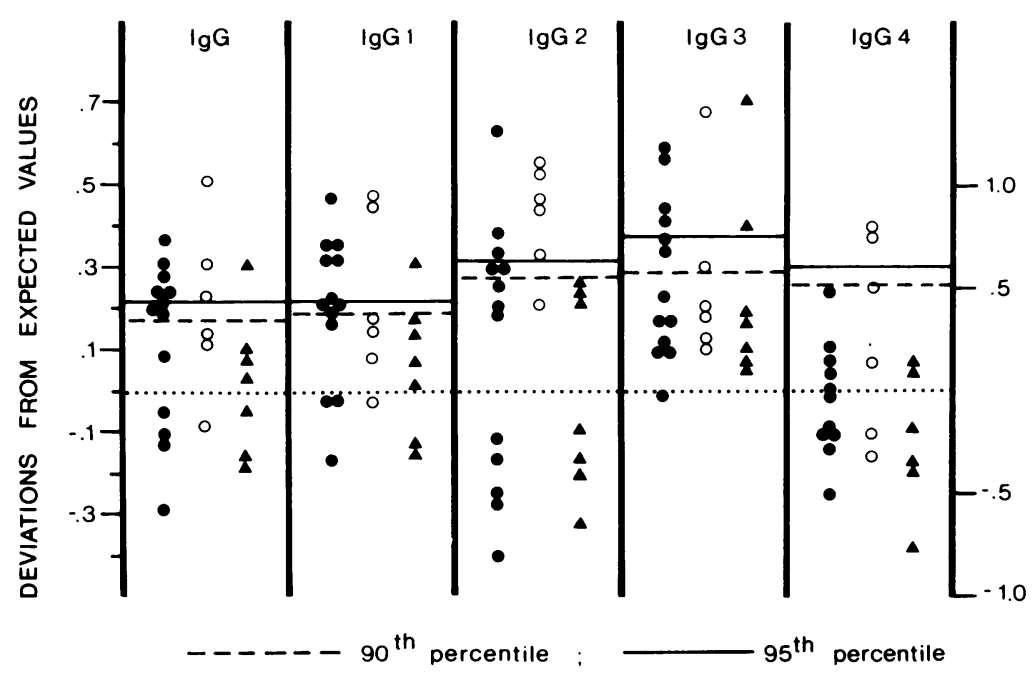

Fig. 3 The JCA patients have been subdivided into three groups with respect to the clinical activity $\mathrm{N}$ of the disease: active disease (O), partial remission $(\mathrm{O})$, remission $(\Delta)$. The values plotted are the deviations from the fitted values (see legend of Fig. 2). The scale on 0 the left vertical axis refers to $\lg G$ and IgG1, IgG2, IgG3 subclass, \& while the scale on the right vertical axis refers to IgG4 subclass only. 
No significant difference in total IgG serum levels was observed between patients with active disease and those in partial remission or in remission, while patients in remission presented significantly lower IgG than patients in partial remission $(p<0.025)$.

\section{Discussion}

In the present study we evaluated IgG subclass levels in sera from 26 patients with JCA. Since subclass concentrations appear to be under genetic control, ${ }^{5}$ in order to have an appropriate control population we compared the data obtained in JCA patients with those of an age matched population of 164 healthy children also evaluated in our laboratory.

We found that IgG1, IgG2, and IgG3 were significantly higher in JCA than in healthy children. IgG3 presented the most striking increase $(\mathrm{p}<0 \cdot 0001)$, followed by IgG1 $(\mathrm{p}<0.002)$, and, to a lesser degree, by $\operatorname{IgG} 2(\mathrm{p}<0.035)$; on the contrary, IgG4 serum concentrations were not significantly different from those of controls. None of the patients had values of IgG1 or IgG3 below the 5th C. One patient presented values of $\mathrm{IgG} 2$ below the 5th $\mathrm{C}$, with normal IgG4 levels, and four patients had levels of IgG4 below the 5th C. No correlation was observed between serum IgG subclass levels and erythrocyte sedimentation rate, sex, disease duration, JCA onset subtype, treatment, presence of antinuclear antibodies, and IgM or IgA serum levels. On the contrary, when patients were divided according to clinical activity of the disease significant increases of IgG2 and IgG4 were observed in patients in partial remission.

The finding of a prevalent restriction of the IgG response to IgG3 and IgG1 suggests a major role for these IgG subclasses in the inflammatory process of JCA. IgG1 and IgG3 indeed have distinct biological properties. ${ }^{5-7}$ The complement components $\mathrm{Clq}$, $\mathrm{C} 3 \mathrm{c}$, and $\mathrm{C} 3 \mathrm{~d}$ bind avidly to IgG1 and IgG3 but less so to IgG2 and fail to bind to IgG4. Peripheral blood lymphocytes have IgG receptors that preferentially bind IgG3; platelets and monocytes have receptors that appear to be specific for IgG1 and IgG3, and polymorphonuclear leucocytes bind IgG1, IgG3, and IgG4.

Our results differ from those observed in adult rheumatoid arthritis (RA), where Shakib and Stanworth found significantly raised serum levels of IgG1 in association with significantly depressed levels of IgG2 and IgG4 and normal levels of IgG3. ${ }^{14}$ The discrepancies could be explained by different patient selection (Shakib's patients were all receiving steroid therapy) or by a real difference in subclass restriction during the inflammatory process in JCA and adult RA. On the other hand, the finding by Hoffman et al of restricted IgG3 production in the IgG synthesised by RA synovial cultures is interesting in relation to the marked increase of serum IgG3 observed in our patients. ${ }^{15}$

Unusual increases of different IgG subclasses have been observed recently in other immune mediated diseases and in some infectious diseases. Studies of IgG subclass deposits in various nephropathies have shown that IgG3 predominates in mesangiocapillary glomerulonephritis ${ }^{16}$ and IgG4 in membranous glomerulonephritis, ${ }^{17}$ while raised IgG1 and IgG3 with low IgG2 and undetectable IgG4 have been reported in children and adults with systemic lupus erythematosus. ${ }^{18}$ Restriction of antibody responses to $\mathrm{IgG} 3$ has been reported in certain viral infections, ${ }^{19}$ and it has been observed that antipolysaccharide antibodies are preferentially IgG2. ${ }^{20}$ The selective increase in IgG subclasses observed in our patients, as in other immune mediated diseases, could thus be a consequence of the immune response to some unknown infectious agent, or could represent a peculiar feature of an intrinsic disorder of immunoregulation.

On the other hand, it is well known that immunodeficiency can be associated with immune mediated diseases. ${ }^{1}$ Recently, selective deficiencies of IgG subclasses have been reported in patients with recurrent infections: in particular, isolated deficiency of IgG4 associated with pyogenic infections, ${ }^{7}$ and IgG2 and IgG4 deficiency in patients with IgA deficiency or ataxia telangiectasia. ${ }^{18}$ One of our patients presented serum levels of IgG2 below the 5 th $\mathrm{C}$, with normal $\mathrm{IgG} 4$, and four had serum levels of IgG4 below the 5th C. However, the latter finding does not allow any definite conclusion since levels of IgG4 are undetectable by radial immunodiffusion in more than $5 \%$ of healthy children. ${ }^{7}$ Moreover, neither the patients nor their relatives presented a history of recurrent infections.

When our patients were divided according to clinical activity of the disease significant increases of IgG2 and IgG4 were observed in children in partial remission, a phase that we might expect to be associated with progressive reduction of the inflammatory process. Since, as discussed before, IgG2 and IgG4 have less inflammatory properties than IgG1 and IgG3, it is tempting to speculate that the above finding may represent a transitory switch of the IgG subclass response associated with the remission inducing process. Follow up studies are now in progress to verify these findings in individual patients, which if confirmed could give insight into the pathogenesis of the disease and represent a useful index for following disease activity and monitoring drug efficacy. It is interesting to note 
that Shakib and Stanworth have reported that in individual adult patients with RA treatment with penicillamine, a remission inducing drug, is accompanied by a selective increase in serum levels of IgG4 and IgG2, ${ }^{6}$ and Garelli et al have described an interesting patient with severe haemolytic anaemia who had IgG1 and IgG3 autoantibodies on her red cells that later switched to IgG2, and IgG4 autoantibodies when the haemolytic crisis subsided. ${ }^{21}$ Finally, Munthe and Natvig found that IgG subclasses in rheumatoid synovial plasma cells of adult patients were present in varying quantities in individual patients, suggesting an individualised selection of subclass response. ${ }^{22}$ Their finding of a predominance of the same subclass in different joints of a given patient suggested that each individual reacts with a certain immunopathological pattern common for all joints but often characteristic for that patient. It may be, however, that the subclass restriction observed in individual patients was related to disease activity.

In conclusion, determination of IgG subclass levels in JCA seems to provide a useful insight into the pathological process operating in the disease. In order to obtain further information follow up studies in individual patients and attempts to show antibody activity within the IgG subclasses are under investigation.

We wish to thank Domenico Zanaboni and Attilio Ascione for skilful technical assistance and Maria Grazia Zambon and Clara Ricotti for kind secretarial assistance. This work was partially supported by CNR Rome and IRCCS Policlinico San Matteo, Pavia.

\section{References}

1 Ansell B M. Rheumatic disorders in childhood. London: Butterworth, 1980.

2 Moore T L, Osborn T D, Weiss T D, et al. Autoantibodies in juvenile arthritis. Semin Arthritis Rheum 1984; 13: 329-36.

3 Cassidy J T, Petty R E, Sullivan D B. Abnormalities in the distribution of serum immunoglobulin concentrations in juvenile rheumatoid arthritis. J Clin Invest 1973; 52: 1931-6.
4 Terry W D, Fahey J L. Subclasses of human 2-globulin based on differences in the heavy polypeptide chains. Science 1964; 146: 400-1.

5 Shakib F, Stanworth D R. Human IgG subclasses in health and disease. (A review) Part I. Ric Clin Lab 1980; 10: 463-79.

6 Shakib F, Stanworth D R. Human IgG subclasses in health and disease. (A review) Part II. Ric Clin Lab 1980; 10: 561-80.

7 Heiner D C. Significance of immunoglobulin G subclasses. Am J Med 1984; 76: 1-6.

8 Brewer E J, Bass J, Baum J, et al. Current proposed revision of JRA criteria. Arthritis Rheum 1977; 20 (suppl 2): 195-9.

9 Pinals R S, Masi A T, Larsen R A. Preliminary criteria for clinical remission in rheumatoid arthritis. Arthritis Rheum 1981; 24: 1308-15.

10 Mancini G, Carbonara A O, Heremans J F. Immunochemical quantitation of antigens by single radial immunodiffusion. Immunochemistry 1965; 2: 235-54.

11 Walker M, Lowe J, Bird P, Ling N R, Jefferis R. Immunogenic and antigenic epitopes of immunoglobulins. Application of monoclonal antibodies to human IgG sub-classes in quantitative assays. In: Pecter H. ed. Protides of the biological fluids. Oxford: Pergamon, 1982; 30: 559-62.

12 Cleveland W S. Robust locally weighted regression and smoothing scatterplots. J Am Statist Assoc 1979; 74: 829-36.

13 Sokal R R, Rohlf F J. Biometry. San Francisco: Freeman, Cooper, 1969.

14 Shakib F, Stanworth D R. IgG subclass composition of rheumatoid arthritic sera and joint fluids. Ann Rheum Dis 1976; 35: 263-6.

15 Hoffman W L, Goldberg M S, Smiley J D. Immunoglobulin G3 subclass production by rheumatoid synovial tissue cultures. $J$ Clin Invest 1982; 69: 136-44.

16 Bannister K M, Howarth G S, Clarkson A R, Woodroffe A J. Glomerular IgG subclass distribution in human glomerulonephritis. Clin Nephrol 1983; 19: 161-5.

17 Doi T, Mayumi M, Kanatu K, Suehiro F, Hamashima Y Distribution of $\mathrm{IgG}$ subclasses in membranous nephropathy. Clin Exp Immunol 1984; 58: 57-62.

18 Oxelius V A. Immunoglobulin $\mathrm{G}(\mathrm{IgG})$ subclasses and human disease. Am J Med 1984; 76: 7-18.

19 Beck D E. Distribution of virus antibody activity among human IgG subclasses. Clin Exp Immunol 1981; 43: 626-32.

20 Riesen W F, Skvaril F, Braun D B. Natural infection of man with group A streptococci: levels, restriction in class, subclass and type and clonal appearance of polysaccharide-groupspecific antibodies. Scand J Immunol 1976; 5: 383-90.

21 Garelli S, Mosconi L, Valbonesi M, Schieppati G, Navassa G. Plasma exchange for a hemolytic crisis due to autoimmune hemolytic anemia of the IgG warm type. Blut 1980; 41: 387-91.

22 Munthe E, Natvig J B. Immunoglobulin classes, subclasses and complexes of IgG rheumatoid factor in rheumatoid plasma cells. Clin Exp Immunol 1972; 12: 55-70. 